\title{
Hydralazine use for the management of hypertensive crises in a medical intensive care unit
}

\author{
Anudeeksha Satheeshkumar BSA, Hunter Atkins MD, Sakolwan Suchartlikitwong MD, \\ Ebtesam Islam MD, PhD
}

\begin{abstract}
Background: Hypertensive crises, including emergent or urgent hypertension, are rare but life-threatening complications of uncontrolled hypertension. Hydralazine is one of several antihypertensive medications available for treatment of hypertensive crises. Major United States guidelines on hypertension recommend conservative use of hydralazine-only for situations of preeclampsia or eclampsia with pregnancy-due to significant adverse effects and unpredictability in the dose response.

Methods: A retrospective chart review was conducted on patients admitted to the medical intensive care unit at University Medical Center in Lubbock, Texas, with urgent or emergent hypertension between January 1, 2017, and June 30, 2017. Demographic information-age and gender-and records of which antihypertensive medication(s) and route used were collected. Systolic blood pressure before and 2 hours after hydralazine administration-and whether it was given after an initial dose of another antihypertensive medication-was recorded. Patient comorbidities and contraindications for use were noted.

Results: Thirty-five patients were included in this study (1 patient result was excluded from certain calculations due to missing data). Mean age of patients was $53.4 \pm 12.5$ years. Range was 22-74 years. Eight patients had initial treatment with hydralazine, and 29 out of 35 patients were given hydralazine when considering combination treatment. IV hydralazine was preferred over PO hydralazine (23 patients vs. 6 patients). Sixteen patients had comorbidities and/or contraindications for hydralazine use, but 12 patients received IV hydralazine and one patient received $P O$ hydralazine.

Conclusion: Hydralazine was not used in a guideline-directed manner in the medical intensive care unit at our hospital. Physicians should regularly evaluate patients for the presence or absence of end-organ damage concurrent with a blood pressure $>180 / 120 \mathrm{mmHg}$ before considering which antihypertensive medication to use. Hydralazine should be reserved for special situations involving pregnancy.
\end{abstract}

Keywords: hydralazine, emergent hypertension, urgent hypertension, intensive medical care unit

\section{INTRODUCTION}

Intensive care management of urgent and emergent hypertension varies across continents. While

\footnotetext{
Corresponding author: Ebtesam Islam

Contact Information: Ebtesam.Islam@ttuhsc.edu

DOI: 10.12746/swrccc.v9i39.855
}

hydralazine is not commonly used in Europe, it now ties with nicardipine as the third most commonly used agent for the treatment of urgent and emergent hypertension in the United States after labetalol and metoprolol. ${ }^{1}$ Several societies have developed guidelines for the treatment of hypertension, including the American College of Cardiology (ACC), American Heart Association (AHA), American College of Physicians $(\mathrm{ACP})$, American Association of Family Physicians 
(AAFP), United States Preventative Services Task Force (USPSTF), Joint National Committee (JNC), and the American Society for Hypertension (ASH). However, most recently, only the 2017 ACC/AHA guidelines and the $2003 \mathrm{JNC} 7$ report have released treatment recommendations specifically addressing the management of urgent and emergent hypertension. ${ }^{2,3}$ The JNC7 report broadly defines hypertensive emergency as occurring when blood pressure is $>180 / 120 \mathrm{mmHg}$ with the presence or indication of end-organ damage. ${ }^{2}$ The 2017 ACC/AHA guidelines specifically address both emergent and urgent hypertension, defining the condition as emergent when blood pressure is $>180 / 120 \mathrm{mmHg}$ with confirmed end-organ damage and as urgent when blood pressure is $>180 / 120 \mathrm{mmHg}$ with the lack of end-organ damage. ${ }^{3}$ The management of hypertensive urgency is often overly aggressive and should be mostly limited to oral medications. ${ }^{2}$ Parenteral treatment should be limited to cases of emergent hypertension. ${ }^{2-5}$

While the guidelines for parenteral treatment are clear (only for emergent hypertension), the use of hydralazine has increased in patients with non-emergent hypertension in whom intra-cranial pressures and/or cardiac status should be considered first. Hydralazine is a potent vasodilator with rapid onset of action that has an unpredictable effect on blood pressure and an unpredictable dose response. ${ }^{6,7}$ Excessive blood pressure reductions have raised concerns about inappropriate hydralazine use. ${ }^{8}$ Although no clinical data have been published supporting this concern at our hospital, IV hydralazine use in a non-guideline directed manner occurs frequently at variance from published guidelines. The only recommendation for IV hydralazine use from both the JNC7 report and the 2017 ACC/AHA guidelines is in emergency situations during pregnancy, such as with eclampsia or pre-eclampsia. ${ }^{2,3}$ This study investigates non-guideline based hydralazine use in our medical ICU to determine both the frequency and clinical outcomes.

\section{Methods}

This is a hypothesis-driven study based on retrospective chart review data with Institutional Review Board approval at Texas Tech University Health Sciences Center in Lubbock, Texas, and with administrative review and approval by University Medical Center (UMC) in Lubbock, Texas. Medical records were identified by a UMC Information Technology department query. Information was collected on patients between the ages of 18-89 who were admitted to the UMC medical intensive care unit for urgent or emergent hypertension between January 1, 2017, and June 30, 2017. Patients older than 89 years or who were admitted for non-urgent or non-emergent hypertension were not included in this study. Basic demographic information included age, gender, and diagnosis. The number of patients diagnosed with either emergent or urgent hypertension was quantified. The charts were reviewed to determine the antihypertensive drug(s) and route used in each patient. The choice of medication(s) and route are then evaluated based on the 2017 ACC/AHA guidelines and the JNC7 report to determine adherence or non-adherence to guidelines. Patients who were treated with intravenous (IV) hydralazine were reviewed for comorbidities, including aortic aneurysms, pulmonary edema, acute ischemic strokes, or myocardial infarctions, which are considered contraindications for use by some guidelines. In addition, the differences in systolic blood pressures pre- and 2 hours post-treatment were recorded to calculate the average drop in systolic blood pressure and to determine the variation in response.

\section{RESULTS}

The average age of 35 patients presenting to the ICU with either urgent or emergent hypertension was $53.4 \pm 12.5$ years (range: $22-74$ ). Nineteen patients $(54.3 \%)$ were women, and $16(45.7 \%)$ were men. Emergent hypertension was present in 19 patients $(54.3 \%)$.

As shown in Table 1, out of 34 patients (1 patient result excluded due to missing data), nicardipine was the drug of choice for initial antihypertensive treatment in 17 patients $(50.0 \%)$. Hydralazine was the second most commonly used drug in 8 patients $(23.5 \%)$, followed by labetalol in 7 (20.6\%), and metoprolol in 2 (5.9\%).

As shown in Table 2, nicardipine remained the primary drug of choice for either subcategory of hypertensive crises. Out of the 19 patients with emergent 
Table 1. Initial Antihypertensive Medication Use

\begin{tabular}{|l|c|c|}
\hline & $\begin{array}{c}\text { Number of Patients } \\
\mathbf{N = 3 4}\end{array}$ & $\begin{array}{c}\text { Percentage of } \\
\text { Patients }\end{array}$ \\
\hline Nicardipine & 17 & $50.0 \%$ \\
\hline Hydralazine & 8 & $23.5 \%$ \\
\hline Labetalol & 7 & $20.6 \%$ \\
\hline Metoprolol & 2 & $5.9 \%$ \\
\hline
\end{tabular}

hypertension, 11 patients (57.9\%) were given nicardipine, $4(21.1 \%)$ were given hydralazine, $3(15.8 \%)$ were given labetalol, and 1 (5.3\%) was given metoprolol. Out of the 15 patients with urgent hypertension, 6 patients $(40.0 \%)$ were given nicardipine, $4(26.7 \%)$ were given hydralazine, $4(26.7 \%)$ were given labetalol, and 1 $(6.7 \%)$ was given metoprolol. Hydralazine was the second most used medication for emergent hypertension and tied for the second most used medication for urgent hypertension with labetalol. Metoprolol was the least administered drug for either emergent or urgent hypertension and was given to only 1 patient in each subcategory.

Even if other antihypertensive medications were used as the initial treatment for either emergent or urgent hypertension, hydralazine was subsequently added to the treatment regimen of 29 out of the 35 patients.

IV hydralazine was more commonly administered over PO hydralazine. Out of 35 patients, IV hydralazine was given to 23 patients $(65.7 \%)$ and $\mathrm{PO}$ hydralazine to 6 patients $(17.1 \%)$. Out of 19 patients with emergent hypertension, 13 patients $(68.4 \%)$ received IV hydralazine and 3 patients $(15.8 \%)$ received $\mathrm{PO}$
Table 2. Initial Antihypertensive Medication Use by Hypertensive Crisis Sub-Category

\begin{tabular}{|l|c|c|}
\hline & $\begin{array}{c}\text { Emergent HTN } \\
\mathbf{N = 1 9}\end{array}$ & $\begin{array}{c}\text { Urgent HTN } \\
\mathbf{N}=\mathbf{1 5}\end{array}$ \\
\hline Nicardipine, $\mathbf{N}=\mathbf{1 7}$ & 11 & 6 \\
& $57.9 \%$ & $40.0 \%$ \\
\hline Hydralazine, $\mathbf{N}=\mathbf{8}$ & 4 & 4 \\
& $21.1 \%$ & $26.7 \%$ \\
\hline Labetalol, $\mathbf{N}=\mathbf{7}$ & 3 & 4 \\
& $15.8 \%$ & $26.7 \%$ \\
\hline Metoprolol, $\mathbf{N}=\mathbf{2}$ & 1 & 1 \\
& $5.3 \%$ & $6.7 \%$ \\
\hline
\end{tabular}

HTN- hypertension.

hydralazine. Out of 16 patients with urgent hypertension, 10 patients $(62.5 \%)$ received IV hydralazine and 3 patients $(18.8 \%)$ received PO hydralazine.

In 29 patients treated with hydralazine, the overall mean SBPs before and after a dose of hydralazine were $182.8 \pm 24.2 \mathrm{mmHg}$ and $152.2 \pm 26.9 \mathrm{mmHg}$ $(P=0.000)$, respectively. Due to missing data, the values for the following blood pressure changes were calculated with 27 patients instead of 29 (Table 3). In 8 patients treated with initial dose of hydralazine, the mean SBP before and after a repeat dose of hydralazine were $202.0 \pm 22.3$ and $164.3 \pm 37.2 \mathrm{mmHg}$ $(P=0.002)$, respectively. In 6 patients with an initial dose of labetalol, the mean SBP before and after a dose of hydralazine were $190.5 \pm 19.2 \mathrm{mmHg}$ and $157.3 \pm 11.8 \mathrm{mmHg}(\mathrm{p}=0.003)$, respectively. In 13 patients treated with an initial dose on nicardipine, the mean SBP before and after a dose of hydralazine

Table 3. Mean Difference in Systolic Blood Pressure Before and After Initial Dose of Various Antihypertensive Medications and Subsequent Dose of Hydralazine

\begin{tabular}{|l|c|c|c|}
\hline & $\begin{array}{c}\text { Initial Dose of } \\
\text { Hydralazine, } \mathbf{N}=\mathbf{8}\end{array}$ & $\begin{array}{c}\text { Initial Dose of } \\
\text { Labetalol, N=6 }\end{array}$ & $\begin{array}{c}\text { Initial Dose of } \\
\text { Nicardipine, } \mathbf{~ = ~ 1 3 ~}\end{array}$ \\
\hline $\begin{array}{l}\text { Systolic BP before } \\
\text { hydralazine (mmHg) }\end{array}$ & $202.0 \pm 22.3$ & $190.5 \pm 19.2$ & $172.3 \pm 18.6$ \\
\hline $\begin{array}{l}\text { Systolic BP after } \\
\text { hydralazine (mmHg) }\end{array}$ & $164.3 \pm 37.2$ & $157.3 \pm 11.8$ & $146.7 \pm 23.6$ \\
\hline Paired samples test p-value & 0.002 & 0.003 & 0.000 \\
\hline
\end{tabular}


Table 4. Clinical Contraindications for the Use of Hydralazine

\begin{tabular}{|l|c|}
\hline & Hydralazine \\
\hline CVA, $\mathbf{N}=\mathbf{1 1}$ & 11 \\
\hline Pulmonary edema, $\mathbf{N}=\mathbf{5}$ & 5 \\
\hline MI, $\mathbf{N}=\mathbf{0}$ & 0 \\
\hline Aortic aneurysm, $\mathbf{N}=\mathbf{0}$ & 0 \\
\hline
\end{tabular}

a-number treated with drug.

CVA- cerebral vascular accident; MI- myocardial infarction.

were $172.3 \pm 18.6 \mathrm{mmHg}$ and $146.7 \mathrm{mmHg} \pm 23.6$ $\mathrm{mmHg}(p=0.000)$, respectively.

Sixteen patients had comorbidities, such as CVA, $\mathrm{MI}$, aortic aneurysms, and pulmonary edema, in addition to their hypertensive crisis (Table 4). Out of 34 patients (1 patient result excluded due to missing data), 11 patients (32.4\%) had CVA, and five patients $(14.7 \%)$ had pulmonary edema. Out of 34 patients, hydralazine use was contraindicated in 16 patients $(47.1 \%)$ based on both their comorbidities and guideline recommendations. Hydralazine use was contraindicated in all 11 patients with CVA and in all 5 patients with pulmonary edema. IV hydralazine was used despite being contraindicated in 12 patients, and $\mathrm{PO}$ hydralazine was used despite being contraindicated in 1 patient.

\section{Discussion}

The purpose of this study was to evaluate the use of hydralazine in patients with either emergent or urgent hypertension in the medical ICU unit of a regional hospital in West Texas. The various antihypertensive drugs administered, the routes of administration, and any patient comorbidities were assessed from a retrospective analysis of clinical data. Hydralazine is considered a direct vasodilator; it decreases the resistance of arterial blood vessels only, which causes an overall decrease in peripheral resistance. ${ }^{9}$ The exact mechanism of action of hydralazine is uncertain. A study by Lipe et al. ${ }^{10}$ noted that the mechanism by which hydralazine relaxes the arterial blood vessels does not involve opposing the receptor sites for common vasoconstrictors or the prevention of extracellular calcium from entering the arteriolar muscle cells. A study by Reidenberg et al. ${ }^{11}$ reported that the halflife of IV and PO hydralazine was similar but differed between genetically "rapid" or "slow" acetylators. For rapid acetylators, the half-life was between 2.2-7.8 hours and for slow acetylators it was between 2.0-5.8 hours. ${ }^{11}$ Hydralazine does not have a strong doseresponse relationship, and, therefore, it can be difficult to predict its overall effect on the patient's blood pressure; additionally, it can cause reflex tachycardia and a reflex BP increase, which is counterproductive. ${ }^{12}$

This study found that hydralazine was often used in a non-guideline directed manner and was the second most commonly ordered antihypertensive medication after nicardipine. Hydralazine is often over-utilized by physicians; in one study, only $2.9 \%$ of 2,189 patients actually qualified for rapid BP reduction with IV hydralazine or other antihypertensives. ${ }^{13,14}$ Campbell et al. ${ }^{8}$ noted that IV hydralazine was often given for cases of asymptomatic hypertension or before a physician evaluated the patient for the presence of end-organ damage first. In the Campbell study, 94 patients were given IV hydralazine when only $4(2 \%)$ were considered to have a hypertensive crisis by current guideline standards. ${ }^{8}$ Using just 1 dose of hydralazine can increase the hospital $12.0 \pm 15.9$ days $(p<0.001),{ }^{14}$ which leaves patients at risk for both nosocomial infections and increased financial burden.

This study has several limitations. First, the sample size was relatively small. This reflects the fact that hypertensive crises are infrequent and occur in approximately $1 \%$ of patients suffering with hypertension. ${ }^{15}$ Second, the information collected depended on information in electronic medical record which may have been incomplete in some patients. In particular, the presence or absence of end-organ damage was not always apparent in each patient. This would have helped accurately classify the patients via current U.S. guideline-based definitions of emergent or urgent hypertension. Third, the method of systolic blood pressure measurement was not recorded or evaluated in this study. Blood pressure measurements can be distorted by a variety of factors such as lack of training of nurses/nurse aides, lack of repeat 
measurements with either a manual or automatic sphygmomanometer, malfunctioning automatic sphygmomanometers, patient anxiety, and even improper positioning of the patient. ${ }^{16}$ Fourth, the pregnancy status of the women treated was not recorded.

The main strength of our study is that it documented that patients were being treated with hydralazine at our medical center in the ICU despite contraindications and in a non-guideline-directed manner, e.g., via a parenteral route for urgent hypertension. In addition, these results demonstrate that hydralazine had a definite effect on systolic blood pressure regardless of the initial antihypertensive medication administered to the patient.

\section{Conclusion}

U.S. guideline-based treatment recommendations vary significantly based on which sub-category of hypertensive crisis and the presence or absence of other acute events, such as acute ischemic stroke, aortic dissection, acute kidney injury, or acute heart failure. ${ }^{17}$ Accurate classification of patients based on US guideline-based definitions of emergent and urgent hypertension would help guide treatment decisions. Since hydralazine is only recommended during aggressive blood reductions in pregnant patients, ${ }^{2,3}$ this study should encourage physicians to reevaluate why/how they are using hydralazine in hypertensive crises and to make more guideline-directed decisions.

Article citation: Satheeshkumar A, Atkins $\mathrm{H}$, Suchartlikitwong S, Islam E. Hydralazine use for the management of hypertensive crises in a medical intensive care unit. Southwest Respiratory and Critical Care Chronicles 2021;9(39):9-14

From: Department of Internal Medicine, Texas Tech University Health Sciences Center, Lubbock, Texas

Submitted: $1 / 1 / 2021$

Accepted: 4/2/2021

Reviewer: Camilo Pena MD

Conflicts of interest: none

This work is licensed under a Creative Commons Attribution-ShareAlike 4.0 International License.

\section{REFERENCES}

1. Salgado DR, Silva E, Vincent J-L. Control of hypertension in the critically ill: a pathophysiological approach. Annals of Intensive Care 2013;3:17. doi:10.1186/2110-5820-3-17.

2. U.S. Department of Health and Human Services, National Institutes of Health, National Heart, Lung, and Blood Institute, National High Blood Pressure Education Program. The Seventh Report of the Joint National Committee on Prevention, Detection, Evaluation, and Treatment of High Blood Pressure [Internet]. NIH Publication. 2004 Aug [cited 2013 Feb 27]; No. 04-5230. First published in JAMA 2003;289(19):25602571. doi:10.1001/jama.289.19.2560. Available from: http:// www.nhlbi.nih.gov/files/docs/guidelines/jnc7full.pdf.

3. Whelton PK, Carey RM, Aronow WS, et al. 2017 ACC/AHA/ AAPA/ABC/ACPM/AGS/APhA/ASH/ASPC/NMA/PCNA Guideline for the Prevention, Detection, Evaluation, and Management of High Blood Pressure in Adults, Journal of the American College of Cardiology (2017), doi: 10.1016/j.jacc. 2017.11.006.

4. Marhefka GD. Acute hypertension: hypertensive urgency and hypertensive emergency. Consultant 2016;56(3):222-232.

5. Weber MA, Schiffrin EL, White WB, et al. Clinical practice guidelines for the management of hypertension in the community. J Clin Hypertens 16:14-26. https://doi.org/10.1111/ jch.12237.

6. Johnston GD. Dose response relationship with antihypertensive drugs. Pharmacol Ther 1992;55:53-93.

7. Ludden TM, Shepherd AMM, McNay JL, Jr, et al. Effect of intravenous dose on hydralazine kinetics after administration. Clin Pharmacol Ther 1983;34:148-52.

8. Campbell P, Baker WL, Bendel SD, et al. Intravenous hydralazine for blood pressure management in the hospitalized patient: its use is often unjustified. J American Society of Hypertension: JASH 2011;5(6):473-477. doi: 10.1016/j. jash.2011.07.002.

9. Laurent S. Antihypertensive drugs. Pharmacological Research 2017;124:116-125.

10. Lipe S, Moulds RFW. In vitro differences between human arteries and veins in their responses to hydralazine. J Pharmacology and Experimental Therapeutics. 1981;217: 204-208.

11. Reidenberg MM, Drayer D, DeMarco AL, et al. Hydralazine elimination in man. Clinical Pharmacology \& Therapeutics 1973:14. doi: 10.1002/cpt1973146970.

12. Johnston GD. Dose-response relationship with antihypertensive drugs. Pharmacology \& Therapeutics 1992:55(1):53-93.

13. Velasco A, Phy M. Hydralazine prn, should we really use it? The Southwest Respiratory and Crit Care Chronicles 2015; 3(10):34. 
14. Weder AB, Erickson S. Treatment of hypertension in the inpatient setting: use of intravenous labetalol and hydralazine. J Clin Hypertens 2010;12:29-33.

15. Varon J. Treatment of acute and severe hypertension: current and newer agents. Drugs 2008;68:283-297.

16. Weder AB. Treating acute hypertension in the hospital: a Lacuna in the guidelines. Hypertension 2011;57(1):18-20. doi:10.1161/HYPERTENSIONAHA.110.164194
17. Satheeshkumar A, Pena C, Nugent K. Current U.S. guidelinebased management strategies for special clinical situations involving hypertensive crises: a narrative review of the literature [published online ahead of print, 2020 Oct 19]. Cardiol Rev 2020;10.1097/CRD.0000000000000364. doi:10.1097/ CRD.0000000000000364. 